\title{
In-Situ TEM Study of Phase Evolution in Individual Battery Materials
}

\author{
Khim Karki ${ }^{1,2}$, Hanlei Zhang ${ }^{1}$, Yiqing Huang ${ }^{1}$, M. Stanley Whittingham ${ }^{1}$, Eric A. Stach ${ }^{2}$ and Guangwen \\ Zhou ${ }^{1}$ \\ 1. NECCES at Binghamton University, Binghamton, USA \\ 2. Brookhaven National Laboratory, Upton, USA
}

There has been significant interest in understanding the mechanism of structural phase evolution occurring in individual battery materials at different state of charge (SOC) levels and at various environmental constraints [1, 2]. For example, a commercially-important $\mathrm{LiNi}_{0.8} \mathrm{Co}_{0.15} \mathrm{Al}_{0.05} \mathrm{O}_{2}$ (NCA) cathode material when over-discharged $(>4.2 \mathrm{~V}$ ) and over-heated can lead to the loss of stoichiometric oxygen from the surface $[1,2]$. The loss of oxygen is detrimental as it can react with inflammable liquid electrolyte and cause thermal runaway. Furthermore, the loss of oxygen is accompanied by the migration/re-ordering of transition metal $(\mathrm{TM})$ ions, which leads to complex phase transformation: $(\mathrm{R} \overline{3} \mathrm{~m}) \rightarrow$ disordered spinel $(\mathrm{Fd} \overline{3} \mathrm{~m}) \rightarrow$ disordered rock salt $(\mathrm{Fm} \overline{3} \mathrm{~m})$. The spinel/rock-salt phase that forms on the surface increases the impedance and degrades the electrochemical activity of the electrode. The local probing of the structural and chemical changes that occur within the individual battery material is thus important. Conventional X-ray techniques are insensitive to localized phase transformation, as they provide only average information from ensemble of particles. In-situ environmental transmission electron microscopy (ETEM) provides a unique platform where individual nanoparticles can be investigated for any morphological, structural or chemical changes, under external stimuli, in real-time [3]. Furthermore, the aberration-corrected ETEM with a differential pumping apparatus allows high spatial resolution of $<0.1$ $\mathrm{nm}$ even in a high-pressure gas environment (e.g., $\left.\mathrm{O}_{2}, \mathrm{H}_{2}\right)$ in the system.

Here, we will discuss our in-situ ETEM approach to correlate the surface oxygen loss with the structural phase transformation when heating the over-charged NCA $(\sim 4.7 \mathrm{~V})$ in various gas environments. Particularly, we utilize in-situ electron energy loss spectroscopy (EELS) for tracking electronic changes in the $\mathrm{O}-\mathrm{K}, \mathrm{Ni}-\mathrm{L}$ and $\mathrm{Co}-\mathrm{L}$ edges at the near-surface region of individual NCA particles from room temperature to $400{ }^{\circ} \mathrm{C}$. The changes in the EELS spectra are correlated with structural changes using highresolution TEM (HRTEM) and fast Fourier transform (FFT) analyses. The over-charged NCA particles are separately introduced to oxidizing $\left(\mathrm{O}_{2}\right)$, vacuum, and reducing $\left(\mathrm{H}_{2}\right)$ conditions and the behavior of oxygen evolution as a function of temperature is evaluated.

Typically, NCA at over-charged conditions can undergo some degree of surface phase transformation to the spinel form even at room temperature [4]. The detailed mechanism of phase changes from layered to complete spinel as a function of SOC will be discussed (Figure 1a). Furthermore, we observed that upon heating [5] individual charged NCA particles under high oxygen partial pressures ( $400 \mathrm{mTorr})$, the onset temperature for the release of lattice oxygen dramatically increases $\left(\sim 300{ }^{\circ} \mathrm{C}\right)$, which can be correlated with the changes in the shift in the $\mathrm{O}-\mathrm{K}$ pre-edge EELS spectra (Figure 1b). However, when charged NCA particles are heated in reducing gas environment (e.g. $\mathrm{H}_{2}$ ), the release of oxygen occurs at the low temperature of around $150{ }^{\circ} \mathrm{C}$ (Figure 1c). In both cases, the severe loss of oxygen at their respective onset temperatures is preceded by bulk phase transformations from predominantly spinel (room temperature) to rock-salt structure (Figure $1, \mathrm{~b}-\mathrm{c}$ ). With the oxygen enrichment, the surface degradation of the particle is minimal at $400{ }^{\circ} \mathrm{C}$. In the reducing environment, reduced metallic domains (Ni) are observed on the parent spinel matrix at $400{ }^{\circ} \mathrm{C}$. The varying degree of oxygen loss observed in gas $\left(\mathrm{O}_{2} \mathrm{vs} . \mathrm{H}_{2}\right)-$ solid 
interface suggests the need for appropriate surface modifications (compatible electrolyte, coatings, etc.) on the NCA particles for their robust electrochemical performance [6].

\section{References:}

[1] Wu et al, Chem Mater. 23 (2011), p. 3953.

[2] Hwang et al, ACS Appl. Mater. Interfaces 6 (2014), p. 15140.

[3] Jinschek, J.R., Chem. Commun. 50 (2014), p. 2696.

[4] Zhang \& Karki et al, J. Phys. Chem. C 121 (2017), p. 1421.

[5] Karki et al, ACS Appl. Mater. Interfaces 8 (2016), p. 27762.

[6] This work is supported as part of the NorthEast Center for Chemical Energy Storage (NECCES), an Energy Frontier Research Center funded by the U.S. Department of Energy, Office of Science, Basic Energy Sciences under Award \# DE-SC0012583. This research used resources of the Center for Functional Nanomaterials, which is a U.S. DOE Office of Science Facility, at Brookhaven National Laboratory under Contract No. DE-SC0012704

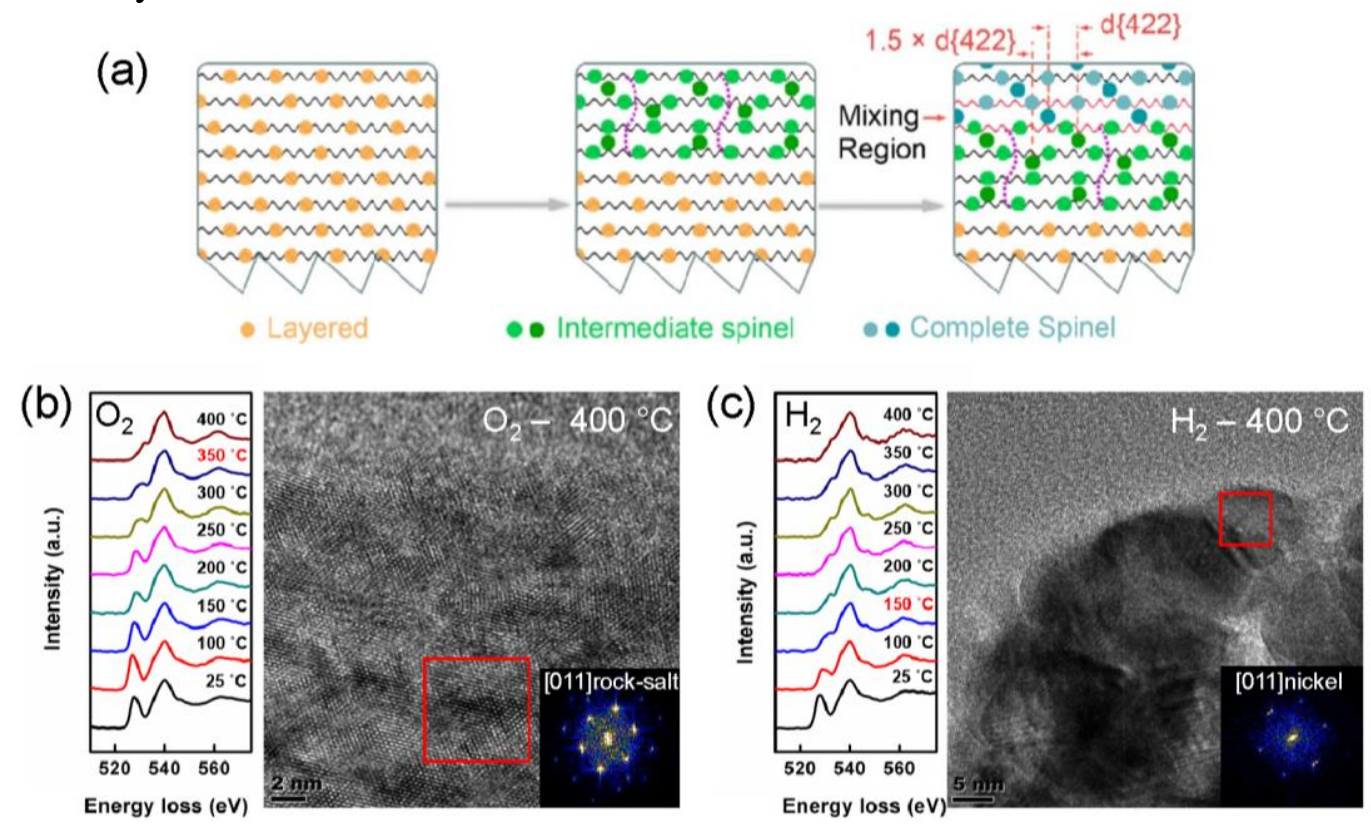

Figure 1. Oxygen evolution and phase transformation of over-charged $(\sim 4.7 \mathrm{~V}) \mathrm{NCA}$ in oxidizing $\left(\mathrm{O}_{2}\right)$ and reducing $\left(\mathrm{H}_{2}\right)$ gas environments. (a) Schematic showing the mechanism of phase evolution from layered $\rightarrow$ spinel at room temperature. (b) O-K EELS intensity profile and corresponding HRTEM image $\left(400{ }^{\circ} \mathrm{C}\right.$ ) at (b) $\mathrm{PO}_{2} \sim 400 \mathrm{mTorr}$ and (c) $\mathrm{PH}_{2} \sim 400 \mathrm{mTorr}$. All spectra in (b) and (c) are normalized to the intensity of main edge. The red boxes in the images represent the areas from which the FFT patterns are extracted. 